\title{
Theory of superconductivity due to Ngai's mechanism in lightly doped $\mathrm{SrTiO}_{3}$
}

\author{
D. E. Kiselov ${ }^{1,2}$ and M. V. Feigel'man ${ }^{2,1}$ \\ ${ }^{1}$ Moscow Institute of Physics and Technology \\ ${ }^{2}$ L.D.Landau Institute for Theoretical Physics, Chernogolovka, Moscow Region, 143432, Russia
}

(Dated: July 6, 2021)

\begin{abstract}
We develop a theory of superconducting pairing in low-density Strontium titanate due to quadratic coupling of electron density to soft transverse optical phonons [1. It leads to static attractive potential between electrons which decay length $l_{\text {eff }}$ that scales inversely with soft optical gap $\omega_{T}$. For low electron densities $n \leq 10^{18} \mathrm{~cm}^{-3}$ attraction between electrons is local and transition temperature $T_{c}$ was found using Ref. [2]. The $T_{c}(n)$ dependence in agreement with experimental data $[3$ for low doping was calculated. Next, we show that suppression of $T_{c}$ by hydrostatic pressure 4 and strong increase of $T_{c}$ due to isotop substitution ${ }^{16} \mathrm{O} \rightarrow{ }^{18} \mathrm{O}$ observed in [5] are explained within our theory.
\end{abstract}

Introduction. Strontium titanate (STO) is a wide-gap band insulator known for more than half a century for its unusual properties related to its proximity to a ferroelectric transition, see Refs [6] for recent reviews. It can be transformed to a very dilute metal by tiny doping, either by oxygen deficiency or by substitution of small portion of $\mathrm{Ti}$ atoms with $\mathrm{Nb}$. The key feature of this metal which makes it very different from majority of various metals and doped semiconductors is that Coulomb interaction is nearly absent due to extremely high lowtemperature static dielectric permeability $\epsilon_{0} \approx 20000$. It results in the huge value of effective Bohr radius, $a_{B} \approx 600 \mathrm{~nm}$, to be compared with normal values $\sim 0.1$ $\mathrm{nm}$ for usual metals. Therefore, Coulomb interaction being the major one in metals normally, appears to be nearly irrelevant for a very dilute STO-based conductor. Suprisingly enough, lighly doped STO becomes superconducting in a wide range of conduction electron densities, $10^{17} \mathrm{~cm}^{-3} \leq n \leq 10^{20} \mathrm{~cm}^{-3}$.

Although first report on superconductivity in STO is dated as early as 1964, see Ref. [9], the mechanism of electron pairing is under active debates till now (examples can be found in Refs. [6 [8]). The key feature of superconductivity in STO (which makes it very different from most of the known superconductors) is due to its very low Fermi energy $E_{F}$ which is much less than Debye energy $\hbar \omega_{D} \approx 100 \mathrm{meV}$. In result, classical theory of superconductivity based upon Migdal-Eliashberg equations [10], cannot be applied here. An attempt to surcumvent this problem was made in Ref. [11 where pairing due to exchange by very soft plasmons was proposed. However, the results obtained for $T_{c}$ were found strongly off the data, if measured value of dielectric constant $\epsilon_{0} \approx 20000$ to be used. Proximity of STO to ferroelectric critical point is surely the crucial feature of this material. The paper [12] pointed out the relevance of this criticality to superconductivity; specifically, they predicted strong increase of $T_{c}$ upon isotop substitution ${ }^{16} O \rightarrow{ }^{18} O$, since the latter is known to produce ferroelectricity when about $\frac{1}{3}$ of $\mathrm{O}$ atoms is replaced by heavy Oxygen [13. Such an effect was indeed observed soon [5]: $35 \%$ substitution ${ }^{16} O \rightarrow{ }^{18} O$ increases $T_{c}$ by factor $\sim 1.5$ and also increases upper critical field nearly twice. An important step forward was made in Ref. [14] which put support to the early idea [1] about relevance of coupling between electron density and two soft transverse optical (TO) phonons, those existence was known since Ref. [15. Namely, Ref. [14] provide arguments based upon analysis of optical absorption spectra in favor of large magnitude of this electron coupling to two TO phonons, of the form $\psi^{\dagger} \psi \mathbf{u}^{2}$, where $\psi$ is the electron annihilation operator and $\mathbf{u}$ is the TO phonon displacement amplitude. Very low gap known for these phonons at low temperatures, $\hbar \omega_{T} \sim 1.5 \mathrm{meV}$, is directly related to large $\epsilon_{0}$ value, $\omega_{T} \propto 1 / \sqrt{\epsilon_{0}}$.

In the present Letter we further develop the ideas proposed in Refs. [1, 14 and use quadratic coupling between electrons and TO phonons as phenomenological input for our theory. We concentrate on the lowest-density limit $n<n_{c 1} \approx 1.5 \cdot 10^{18} \mathrm{~cm}^{-3}$ where single-band Fermiliquid in realized 3, and demonstrate that electronelectron interaction mediated via two TO phonons leads to consistent description of superconducting $T_{c}(n)$ evolution with $n$, Ref. [3], and of its giant isotop effect [5]. Pairing by two-phonon exchange differs a lot from usual single-phonon exchange but leads to a simple picture: electrons attract each other via static potential which decays with a distance as $-V(r) \propto r^{-3} e^{-2 r / l_{0}}$, where $l_{0}=s / \omega_{T O} \approx 3.3 \mathrm{~nm}$ is the characteristic length related to soft polarization TO mode; for the velocity of the TO mode we use $s=7.5 \cdot 10^{5} \mathrm{~cm} / \mathrm{s}$, see [16. At low electron densities we consider, $k_{F} l_{0} / 2<0.6$ and electron-electron scattering can be considered short-range. Frequency dispersion of e-e scattering occurs then at the energy scale $\epsilon \geq \omega_{T}$, and it is relatively weak in the low-density region, since $E_{F}(n)$ does not exceed $\omega_{T}$ considerably.

Superconductivity in a bulk Fermi-gas with local attraction was studied theoretically long ago by Gor'kov and Melik-Barhudarov [2], see also more recent paper [19. They found expression for $T_{c}$ similar to the one known for usual BCS theory, with the major exception that Debye energy is replaced by Fermi energy in 
the prefactor, $T_{c} \approx 0.27 E_{F} e^{-1 / \lambda_{0}}$. Dimensionless coupling constant $\lambda_{0}=\nu_{0} V_{0}$, where $V_{0}$ is the renormalized electron-electron scattering potential in the $l=0$ scattering state (s-wave), and $\nu_{0}$ is the density of states at Fermilevel per single spin projection. Below we demonstrate that attractive short-range potential does indeed appear due to two TO phonon exchange, and calculate $T_{c}$ as function electron density $n$. Then we extend our results to the case of partial isotopic substitution ${ }^{16} O \rightarrow{ }^{18} O$, which reduces $\omega_{T}$ and thus increases $V_{0}$ and $T_{c}$.

Electron-electron interaction mediated by a pair of TO phonons. We start with the action for coupled electronphonon system close to ferroelectric transition:

$$
\begin{aligned}
S & =S_{e}^{(0)}+S_{p h}^{(0)}+S_{\mathrm{int}} \\
S_{p h}^{(0)} & =\frac{\rho_{m}}{2} \int d^{3} x d t\left[\dot{u}_{\alpha}^{2}-s^{2}\left(\nabla_{\beta} u_{\alpha}\right)^{2}-\omega_{T}^{2} u_{\alpha}^{2}\right] \\
S_{\mathrm{int}} & =-g \rho_{m} \int d^{3} x d t(\bar{\psi} \psi) u_{\alpha}^{2}
\end{aligned}
$$

and $S_{e}^{(0)}$ is just the action of free electron gas with effective mass $m_{e}=1.8 m_{0}$, according to the data from Ref. 20] for low electron densities in STO, $m_{0}$ being free electron mass. Here $u_{\alpha}$ is displacement coordinate for TO soft optical phonon, $\psi(x)$ is the electron field operator and $\rho_{m}=5.11 \mathrm{~g} / \mathrm{cm}^{3}$ is the mass density of STO. The action $S_{p h}^{(0)}$ describes long-wave-length TO phonons with momenta $q \ll K_{B Z}$, where $K_{B Z}$ is the boundary of the Brilliuen zone. Whenever high- $q$ cut-off will be needed in the further calculations, we introduce it by using the simplest TO phonon lattice spectrum of the form appropriate for cubic lattice, with the BZ including $p_{x, y, z} \in\left(-\frac{\pi}{a}, \frac{\pi}{a}\right)$ :

$$
\omega^{2}(p)=\omega_{T}^{2}+\frac{4 s^{2}}{a^{2}}\left(\sin ^{2} \frac{p_{x} a}{2}+\sin ^{2} \frac{p_{y} a}{2}+\sin ^{2} \frac{p_{z} a}{2}\right)
$$

where $a \approx 0.4 \mathrm{~nm}$ is the lattice spacing. The coupling constant $g$ in Eq.(1) has natural dimension Length ${ }^{3} /$ Time $^{2}$; we represent it in the form

$$
g=\lambda a^{3} \omega_{L}^{2}
$$

where $\hbar \omega_{L} \approx 0.1 \mathrm{eV}$ is the largest longitidinal optical gap of STO, and $\lambda \sim 1$ is the dimensionless coupling constant of the problem. In a recent paper 21 the same type of electron coupling to TO phonons was employed to study theoretically high-temperature transport properties of lightly doped STO; comparison of this theory predictions with the data 22 provide the value of the coupling constant $\lambda \approx 0.9$.

Static interacting potential between two electrons can be obtained from the action (1) by integrating out the phonons; the corresponding diagram is shown in Fig 1 .

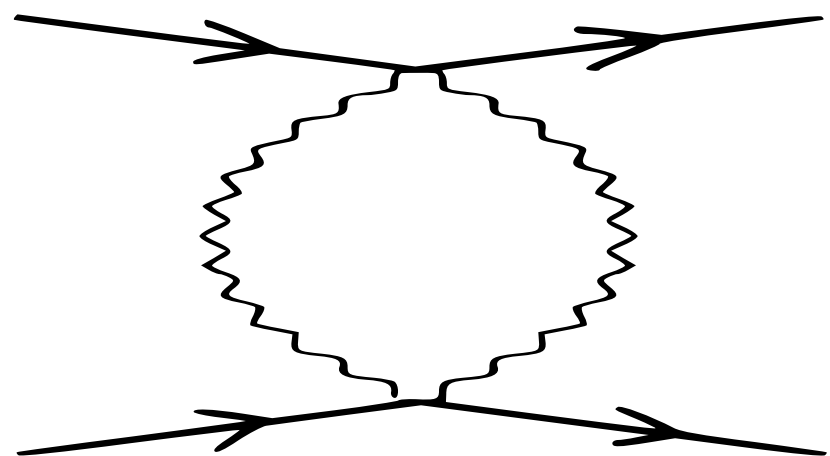

FIG. 1: The diagram corresponding to the static interacting potential between electrons. Solid (wavy) lines denote electron (phonon) Green functions correspondingly.

Its analytical expression in Fourrier space is

$V(\omega, q)=-4 g^{2} \rho_{m}^{2} \int \frac{d \epsilon}{2 \pi} \int_{B Z} \frac{d^{3} p}{(2 \pi)^{3}} D_{0}(\epsilon, \mathbf{p}+\mathbf{q}) D_{0}(\omega-\epsilon, \mathbf{p})$

where momentum integration goes over simple cubic Brilliuen zone and phonon Green function $D_{0}$ is given, in the imaginary-time formalism, by

$$
D_{0}(\epsilon, \mathbf{p})=\frac{1}{\rho_{m}}\left(\epsilon^{2}+\omega_{T}^{2}+\omega^{2}(p)\right)^{-1}
$$

The minus sign in Eq. (4) is the most important our observation; it appears since we deal here with the 2nd order of expansion over virtual phonons. Factor 4 in Eq.(4) comes about due to two variants of pairing in the average $\left\langle u^{2}(0) u^{2}(x)\right\rangle$, and two independent polarizations of TO phonons. We start from the static interaction potential $V(0, q)$, and will discuss the issue of frequency dispersion later on.

Integration over $d \epsilon$ is trivial; using Eqs. 2234 we come to

$$
V(0,0)=-\hbar \lambda^{2} \frac{\left(a^{3} \omega_{L}^{2}\right)^{2}}{s^{3}} \int_{\tilde{B Z}} \frac{d^{3} \tilde{p}}{(2 \pi)^{3}} \frac{1}{\left[\kappa^{2}+a^{2} \omega^{2}(\tilde{p}) / s^{2}\right]^{3 / 2}}
$$

where $\tilde{\mathbf{p}}=a \mathbf{p}$ is dimensionless momentum and $\kappa=$ $a \omega_{T} / s=a / l_{0}$. Dimensionless integral in Eq.(6) is equal to $J / 2 \pi^{2}$, where $J$ is logarithmically large due to smallness of $\kappa \approx 0.12$. We compute $J$ numerically, and find

$$
V(0,0)=-\hbar \lambda^{2} \frac{\left(a^{3} \omega_{L}^{2}\right)^{2}}{2 \pi^{2} s^{3}} \ln \frac{\eta}{\kappa} \equiv-W \ln \frac{\eta}{\kappa}
$$

where $\eta \approx 5.76$. To estimate $T_{c}$ below we will need to know $V(0, q)$ more accurately, up to the term $\sim q^{2}$. We find this additional term by expansion over $q$ in the integral (4). The resulting integral converges fast at large $p$, so no lattice cut-off is needed. Finaly we get:

$$
-V(0, q)=W\left[\ln \frac{\eta}{\kappa}-\frac{\left(q l_{0}\right)^{2}}{12}\right]
$$


Note that dependence on $q$ is relatively weak, as well as dependence of $V(\epsilon, q)$ on $\epsilon$ (to be discussed below); the reason is that major (logarithmic) contribution to the integral in Eq.(4) comes from TO phonons with momenta $p$ in a broad range $k_{F} \leq p \leq \pi / a$. For the same reason the effects of renormalization of phonon spectrum due to interaction with electrons are weak at low concentrations $n_{e} \leq 1.5 \cdot 10^{18} \mathrm{~cm}^{-3}$; we will discuss these effects later on. For completeness, we provide the e-e potential in coordinate space at $r \geq a\left(K_{1}\right.$ is the MacDonald function):

$$
-V(r)=\frac{W}{2 \pi l_{0} r^{2}} K_{1}\left(\frac{2 r}{l_{0}}\right)
$$

Superconducting transition temperature. Attractive e-e interaction defined by Eqs. 89 decays exponentially at $r>l_{0} / 2$ so it can be considered as nearly local in the range of electron densities $n<n_{c 1}=1.5 \cdot 10^{18} \mathrm{~cm}^{-3}$. Then we can employ the theory 2 for superconductivity in a Fermi-gas with local instantenous attraction. The result 2 for $T_{c}$ is

$$
T_{c}=\zeta E_{F} \exp \left(-\frac{1}{\nu_{0} V_{0}}\right) \quad \zeta=\frac{e^{C}}{\pi}\left(\frac{2}{e}\right)^{7 / 3} \approx 0.27
$$

where $\nu_{0}=m_{e} k_{F} / 2 \pi^{2} \hbar^{2}$ is the DoS per one spin projection and $V_{0}$ is the $l=0$ harmonics of the pairing potential (8) evaluated at the Fermi-surface. Assuming FS to be spherical (which is good approximation for STO at low densities), we find (here $\theta$ is the azimutal angle at the FS, so $\left.q=2 k_{F} \sin \frac{\theta}{2}\right)$ :

$$
V_{0}=\frac{1}{2} \int_{0}^{\pi}|V(0, q)| \sin \theta d \theta=W\left(\ln \frac{\eta}{\kappa}-\frac{1}{6} k_{F}^{2} l_{0}^{2}\right)
$$

The plot of $T_{c}(n)$ dependence which follows from Eqs. (10 11), together with definition of $W$ in Eq.(7), is shown in Fig. 2 together with the data from Ref. 3].

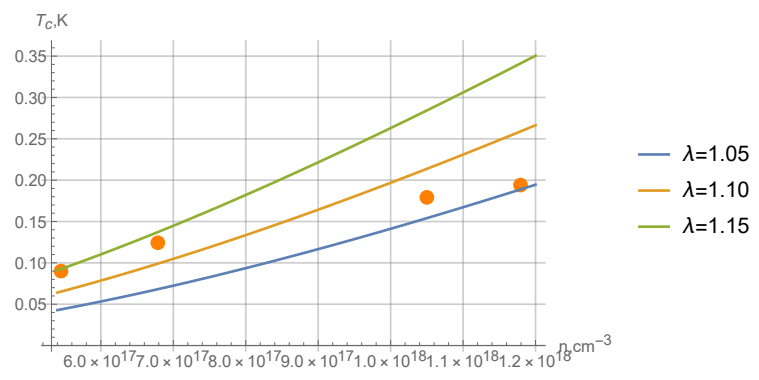

FIG. 2: Crtitical temperature as function of conduction electron density for several values of electron-phonon interaction constant $\lambda$. Orange points represent experimental data from Ref. 3 .

The choice of electron-phonon coupling constant $\lambda=$ 1.1 provides the best correspondence with the data. Some descrepancy is still present, and it will be discussed below.

Suppression of $T_{c}$ by hydrostatic pressure. Relevance of STO proximity to ferroelectric critical point to the origin of superconductivity was extensively discussed in Ref. 4. In particular, they present data on the effect of hydrostatic pressure upon $T_{c}$ and upon dielectric constant $\epsilon$, see Fig.2 in Ref. 4] which demonstrate that decrease of $\epsilon$ leads to sharp fall of $T_{c}$. According to the standard Lyddane-Sachs-Teller relation, $\epsilon(P) / \epsilon=\left(\omega_{T} / \omega_{T}(P)\right)^{2}$ where subsript $P$ stands for pressure-modified values. Using the data from Fig.2b of Ref. 4], we calculated, following our theory, $T_{c}(P)$ for our largest electron density $n_{e}=1.2 \cdot 10^{18} \mathrm{~cm}^{-3}$. The result it present in Fig. 3 .

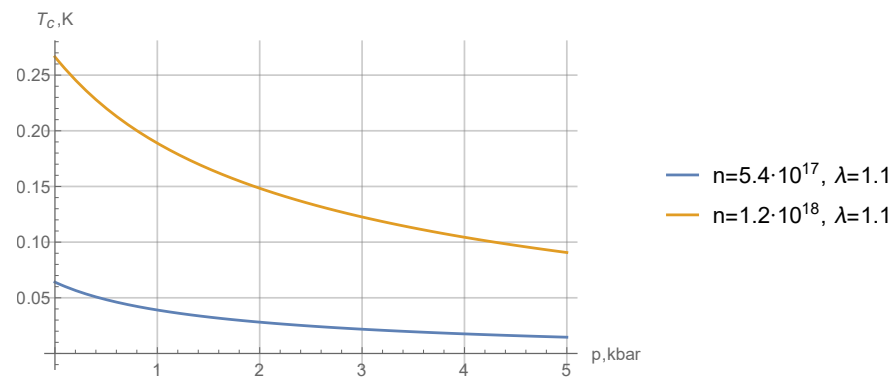

FIG. 3: Plot for crtitical temperature-pressure dependence for two values of electron density indicated in the plot, obtained with $\lambda=1.1$ and $\omega_{T}(P)$ dependence extracted from the data of Ref. [4.

Accurate comparison of our prediction for $T_{c}$ suppression with the corresponding data from Ref. 44 is not possible since they studied the sample with much higher electron density $n=3.4 \cdot 10^{19} \mathrm{~cm}^{-3}$, the overall trend is similar. Our theory predicts a bit smaller suppression effect: factor 2.5 between $P=0$ and $P=4 \mathrm{KBar}$, while experiment 4] provides supprssion factor close to 4 , at the electron density which is 30 times higher. One cannot exclude that hydrostatic pressure may decrease a little the coupling constant $\lambda$, which would lead to additional suppression of $T_{c}$, not accounted in our results in Fig 3 .

Isotopic enhancement of $T_{c}$. In classical weak-coupling superconductors with phonon mechanism of e-e attraction, isotopic substitution of some part of atoms by their heavier isotops leads usually to weak suppression of $T_{c}$ with increase of the typical atom mass $M$. The reason is that $T_{c}=1.13 \omega_{D} e^{-1 / \lambda_{\text {eff }}}$ is proportional to the Debye frequence $\omega_{D} \propto 1 / \sqrt{M}$, while effective coupling constant $\lambda_{\text {eff }}$ is independent on $\omega_{D}$; the latter statement is not entirely evident, but it follows from Eliashberg theory of phonon-coupled superconductors [10]. Recent experimental data present in Ref. [5] demonstrate sharp departure from the usual behaviour: substitution of $35 \%$ of Oxygen atoms ${ }^{16} \mathrm{O}$ by their heavy isotop ${ }^{18} \mathrm{O}$ resulted in a factor $\approx 1.5$ raise of $T_{c}$ for the whole range of studied electron densities, $4 \cdot 10^{18} \mathrm{~cm}^{-3}<n<10^{20} \mathrm{~cm}^{-3}$. 
Another set of data demonstrating the same effect (for higher electron densities) can be found in Ref. 23.

To explain this giant positive isotop effect, we note that under such an isotop substitution, insulating STO becomes a ferroelectric [13. It means that in the isotopmodified material the TO gap $\omega_{T} \rightarrow 0$. To take it into account within our theory of superconductivity in STO, we need just to calculate the ampltiude $V_{0}$ at $\omega_{T}=0$. Now logarithmic integral in Eq. (6) diverges at the lower limit, while for $V(q)$ we find, instead of Eq.8), the following result:

$$
\tilde{V}(q)=-W \ln \frac{\eta e}{q a}
$$

Calculating the $l=0$ scattering amplitude like in Eq.(11), we find

$$
\tilde{V}_{0}=-W\left(\ln \frac{\eta e^{3 / 2}}{2 k_{F} a}\right)
$$

We calculated, using Eqs.13) and (11), the ratio of transition temperatures for isotop-substituted and native STO at the two values of carrier concentrations, $n_{\text {low }}=5.4 \cdot 10^{17} \mathrm{~cm}^{-3}$ and $n_{\text {high }}=1.2 \cdot 10^{18} \mathrm{~cm}^{-3}$ and found

$$
\left(\frac{\tilde{T}_{c}}{T_{c}}\right)_{n_{\text {low }}}=2.5 \quad\left(\frac{\tilde{T}_{c}}{T_{c}}\right)_{n_{\mathrm{high}}}=1.8
$$

The data [5] provide increase of $T_{c}$ by factor 1.5 at even higher concentrations, starting from $n=4 \cdot 10^{18} \mathrm{~cm}^{-3}$, which seems to be quite consistent with our results in Eq. 14. For a weaker isotopic substitution $\omega_{T}$ can be only partially suppressed, so the enhancement of $T_{c}$ will be smaller. We calculated transition temperature $\tilde{T}_{c}$ as function of partially suppressed TO gap $\omega_{T}$. It was done by means of numerical integration starting from $\omega=0$ version of Eq.(4); the result is present in Fig. 4 and can serve as a prediction for future experiments with isotopsubstituted STO.

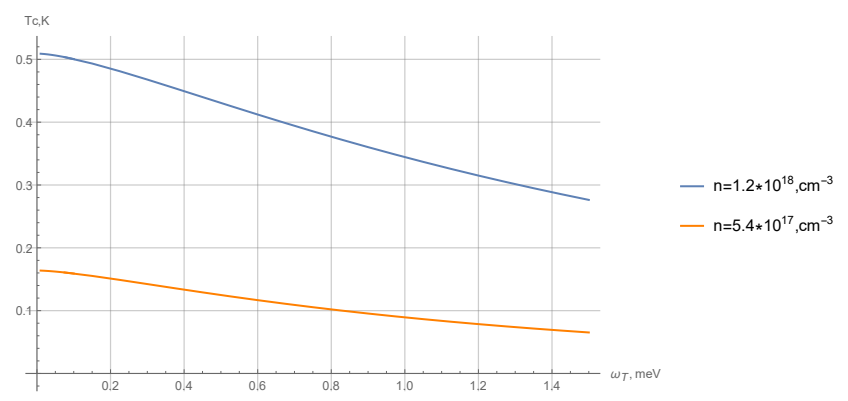

FIG. 4: Crtitical temperature as function of the TO phonon gap $\omega_{T}$ for two values of the electron density.

Note that relative enhancement of the upper critical field $H_{c 2}$ observed in Ref. [5] due to isotop substitution is higher than the corresponding increase of $T_{c}$ - roughly it is a factor 2 instead of 1.5. We expect it to be related with additional disorder which accompanies partial isotopic substitution. Indeed, normal-state resistance of isotopsubstituted samples is enhanced, see Ref. 23.

Conclusions. We developed a theory able to predict superconducting transition temperature in lightly doped STO as function of conduction electron density $n \leq n_{c 1}=1.5 \cdot 10^{18} \mathrm{~cm}^{-3}$; the theory has single fiting parameter $\lambda$ which determines electron coupling to a pair of TO phonons. Comparision with the data for $T_{c}(n)$ from Ref. 3] selects the optimal value this parameter $\lambda=1.1$, which is close to the value 0.9 found by totally different method in Ref. [21]. We note that the estimate for effective strength of e-e attraction $\lambda_{2 \mathrm{ph}} \approx 0.28$ found in Ref. [14 for much higher values of $n_{e}$ does not include large logarithmic factor we discovered, see Eq. (7). It is due to integration over large phase volume of two virtual TO phonons. Our theory is in good qualitative agreement with experiments on $T_{c}$ effects due to isotopic substitution [5, 23] and hydrostatic pressure [4].

The limitation of low densities used in our theory was used, in the first place, in order to concentrate on the simplest situation of a single band filled by conduction electrons, while at higher $n$ second band starts to be filled [3] and more involved calculations are necessary. There are few effects which we neglected so far while they are present, in princple, in the single-band problem as well. First of them is the renormalization of effective phonon gap $\omega_{T}$ due to the presence of the coupling to electrons. Apparently it is given by replacement $\omega_{T}^{2} \rightarrow \omega_{T}^{2}+2 g n$ which could lead to considerable effect even in our range of $n$. However, comparison with the data 24] for $n>10^{19} \mathrm{~cm}^{-3}$ shows much smaller increase of the gap, compatible with $\Delta \omega_{T}^{2} \approx 0.3 \mathrm{gn}$, which does not lead to any noticeable effect at $n \leq n_{c 1}$. The difference between the data from Ref. 24] and naive expectations is probably due to the fact that TO phonons interact both with conduction electrons and with ion defects $(\mathrm{O}$ deficiency or $\mathrm{Nb}$ substitution), and these defects partially suppresses the increase of the gap caused by electrons.

The second relevant effect is due to frequencydependence of the effective e-e interaction, Eq. (4). Indeed, static approximation is definitely fine when $E_{F}<$ $\hbar \omega_{T}$. In fact, $E_{F}$ starts to exceed $\hbar \omega_{T}$ already at $n>7 \cdot 10^{17} \mathrm{~cm}^{-3}$. However, analysis of Eq. (4) shows that $V(\epsilon, 0)-V(0,0) \approx W \frac{\epsilon^{2}}{3 \omega_{T}^{2}}$ while $-V(0,0)=W \ln \frac{\eta}{\kappa} \approx$ $3.8 \mathrm{~W}$. Thus we expect that retardation effects are relatively minor up to $n_{c 1}$, while at higher $n$ the theory [2] should be augmented to take them into account; the same is needed for the accurate analysis of $T_{c}$ enhancement due to isotop substitution leading to $\omega_{T}$ suppression.

A separate comment refers to the preprint 25 ] where tendency to superconductivity was observed down to extremely low electron densities $3.5 \cdot 10^{16} \mathrm{~cm}^{-3}$, with mid- 
point $T_{c} \approx 50-70 \mathrm{mK}$ weakly dependent on $n$ below $10^{18} \mathrm{~cm}^{-3}$. Simultaneously, the absence of diamagnetic shielding expected for any bulk superconducting state was reported 25. We expect that the origin of both these observations is related with large-scale variations of Oxygen vacancy densities over the sample; then resistivitydefined $T_{c}$ is controlled by the regions of larger electron densities, while diamagnetic currents are very weak due to percolative nature of coupling on large scale.

The absence of Coulomb interaction in doped STO makes it rare representative of a superconductor where universal effect of $T_{c}$ suppression by disorder [26] is not operating. Moreover, it may demonstrate the opposite effect of $T_{c}$ enhancement by strong disorder, predicted earlier in bulk 27] and 2D 28] materials with suppressed Coulomb interaction. It might be possible to reach the necessary range of strong disorder, $k_{F} l \sim 1$, by heavydose electron irradiation of STO crystal, along the lines of Ref. [29]. However, such an irradiation may lead to increase of the gap $\omega_{T}$ and thus decrease of effective attraction, so $\omega_{T}$ dependence on irradiation should be controlled.

Acknowledgments We thank Kamran Behnia, Clément Collignon, Benoit Fauqué and Marcin Konczykowski for useful discussions of experimental aspects of STO. We are grateful to Alexei Ioselevich, Khachatur Nazaryan and Igor Poboiko for their comments on theory side. This research was supported by the RSCF grant \# 21-12-00104.

[1] K. L. Ngai, Phys. Rev. Lett. 32, 215 (1974)

[2] L.P.Gor'kov and T.K.Melik-Barkhudarov, Sov.Phys. JETP 40, 1452-1458 (1961)

[3] Xiao Lin, German Bridoux, Adrien Gourgout, Gabriel Seyfarth, Steffen Kramer, Marc Nardone, Benoit Fauque, and Kamran Behnia, Phys. Rev. Lett. 112, 207002 (2014).

[4] C. Enderlein, J. Ferreira de Oliveira, D. A. Tompsett, E. Baggio Saitovitch, S. S. Saxena, G. G. Lonzarich and S. E. Rowley, "Superconductivity mediated by polar modes in ferroelectric metals", Nature Comm. 11, 4852 (2020)

[5] A. Stucky, G. W. Scheerer, Z. Ren, D. Jaccard, J.-M. Poumirol, C. Barreteau, E. Giannini and D. van der Marel, Scientific Reports, 6:37582 (2016)

[6] Maria N.Gastiasoro, Jonathan Ruhman and Rafael M.Fernandes, "Superconductivity in dilute SrTiO: a review", Annals of Physics, vol. 417, p. 168107 (2020)

[7] Clement Collignon, Xiao Lin, Carl Willem Rischau, Beno Fauque, and Kamran Behnia, "Metallicity and Superconductivity in Doped Strontium Titanate", Annual Review of Condensed Matter Physics, vol. 10, p. 25 (2019)

[8] Gernot Scheerer, Margherita Boselli, Dorota Pulmannova, Carl Willem Rischau , Adrien Waelchli, Stefano Gariglio, Enrico Giannini , Dirk van der Marel and JeanMarc Triscone, "Ferroelectricity, Superconductivity,and SrTiO3 - Passions of K.A. Muller", Condens. Matter, 5, 60 (2020);
[9] J. F. Schooley, W. R. Hosler, and M. L. Cohen, "Superconductivity in Semiconducting SrTiO3", Phys. Rev.Lett. 12, 474 (1964).

[10] G.M. Eliashberg, "Interactions between Electrons and Lattice Vibrations in a Superconductor", Zh. Eksperim. i Teor. Fiz. 38, 966 (1960); Sov. Phys. JETP 11, 696-702 (1960).

[11] J. Ruhman and P.A. Lee, Phys. Rev. B 94, 224515 (2016)

[12] Jonathan M. Edge, Yaron Kedem, Ulrich Aschauer, Nicola A. Spaldin, and Alexander V. Balatsky, Phys Rev. Lett. 115, 247002 (2015).

[13] M. Itoh, R. Wang, Y. Inaguma, T. Yamaguchi, Y-J. Shan, and T. Nakamura, "Ferroelectricity Induced by Oxygen Isotope Exchange in Strontium Titanate Perovskite", Phys. Rev. Lett. 82, 3540 (1999).

[14] D. van der Marel, F. Barantani, and C. W. Rischau Phys. Rev. Research 1, 013003 (2019)

[15] Y. Yamada and G. Shirane, "Neutron scattering and nature of the soft optical phonon in SrTiO3". J. Phys. Soc. Jpn. 26, 396 (1969).

[16] The value of $s \approx 7.5 \cdot 10^{5} \mathrm{~cm} / \mathrm{s}$ follows from the data in Refs. 15, 17] and from recent measurements [18.

[17] E. Courtens, G. Coddens, B. Hennion, B. Hehlen, J. Pelous and R. Vacher, "Phonon Anomalies in SrTiO3 in the Quantum Paraelectric Regime", Phys. Scr. T 49, 430 (1993).

[18] B.Fauqué, private communications.

[19] Lev P. Gor'kov, "Superconducting transition temperature: Interacting Fermi gas and phonon mechanisms in the nonadiabatic regime", Phys. Rev. B 93, 054517 (2016)

[20] X. Lin, Z. Zhu, B. Fauque, and K. Behnia, Phys. Rev. X 3, 021002 (2013).

[21] Kh. G. Nazaryan and M. V. Feigel'man, arXiv:2103.11425

[22] Clement Collignon, Phillipe Bourges, Benoit Fauque, and Kamran Behnia, "Heavy non-degenerate electrons in doped strontium titanate", Phys. Rev. X 10, 031025 (2020)

[23] Tomioka, Y., Shirakawa, N., Shibuya, K. Inoue, I. H. "Enhanced superconductivity close to a non-magnetic quantum critical point in electron-doped strontium titanate", Nat. Commun. 10, 738 (2019).

[24] J. L. M. van Mechelen, D. van der Marel, C. Grimaldi, A. B. Kuzmenko, N. P. Armitage, N. Reyren, H. Hagemann, and I. I. Mazin, "Electron-Phonon Interaction and Charge Carrier Mass Enhancement in SrTiO3", PRL 100, 226403 (2008)

[25] Terence M. Bretz-Sullivan at al,"Superconductivity in the dilute single band limit in reduced Strontium Titanate ", arXiv:1904.03121 2

[26] A. M. Finkelstein, JETP Lett. 45, 46 (1987); Sov. Sci. Rev., Sect. A 14, 1 (1990); Physica B 197, 636 (1994).

[27] M. V. Feigelman et al., Phys. Rev. Lett. 98, 027001 (2007); Ann. Phys. (N.Y.) 325, 1390 (2010).

[28] I. S. Burmistrov, I. V. Gornyi, and A. D. Mirlin, "Enhancement of the Critical Temperature of Superconductors by Anderson Localization", Phys. Rev. Lett. 108, 017002 (2012)

[29] Xiao Lin, Carl Willem Rischau, Cornelis J. van der Beek, Benoit Fauqué, and Kamran Behnia, "S-wave Superconductivity in Optimally Doped SrTiNbO3 Unveiled by Electron Irradiation", Phys. Rev. B 92, 174504 (2015). 